\title{
Evidence of Microbiome-Drug Interaction between the Antimalarial Lumefantrine and Gut Microbiota in Mice
}

\begin{abstract}
Matthew M. Ippolito, ${ }^{1,2,3,4 *} \dagger$ Joshua E. Denny, ${ }^{5} \dagger$ Elizabeth Nenortas, ${ }^{1,2,3}$ Theresa A. Shapiro, ${ }^{1,2,4}$ and Nathan W. Schmidt ${ }^{5 \star}$ ${ }^{1}$ Division of Clinical Pharmacology, Department of Medicine, Johns Hopkins University School of Medicine, Baltimore, Maryland; ${ }^{2}$ Department of Pharmacology and Molecular Sciences, Johns Hopkins University School of Medicine, Baltimore, Maryland; ${ }^{3}$ Division of Infectious Diseases, Department of Medicine, Johns Hopkins University School of Medicine, Baltimore, Maryland; ${ }^{4}$ Johns Hopkins Malaria Research Institute, Johns Hopkins University Bloomberg School of Public Health, Baltimore, Maryland; ${ }^{5}$ Department of Microbiology and Immunology, University of
\end{abstract} Louisville, Louisville, Kentucky

\begin{abstract}
The antimalarial drug lumefantrine exhibits erratic pharmacokinetics. Intersubject variability might be attributed, in part, to differences in gut microbiome-mediated drug metabolism. We assessed lumefantrine disposition in healthy mice stratified by enterotype to explore associations between the gut microbiota and lumefantrine pharmacokinetics. Gut microbiota enterotypes were classified according to abundance and diversity indices from 16S rRNA sequencing. Pharmacokinetic parameters were computed using noncompartmental analysis. Two distinct enterotypes were identified. Maximal concentration $\left(C_{\text {max }}\right)$ and total drug exposure measured as the area under the drug concentration-time curve $\left(\mathrm{AUC}_{0-24}\right)$ differed significantly between the groups. The mean and standard deviation of $C_{\max }$ were $660 \pm 220 \mathrm{ng} / \mathrm{mL}$ versus $390 \pm 59 \mathrm{ng} / \mathrm{mL}(P=0.02)$, and $\mathrm{AUC}_{0-24}$ was $9,600 \pm 2,800$ versus $5,800 \pm 810 \mathrm{ng} \times \mathrm{h} / \mathrm{mL}(P=0.01)$. In healthy mice intragastrically dosed with the antimalarial drug lumefantrine in combination with artemether, lumefantrine exposure was associated with gut bacterial community structure. Studies of xenobiotic-microbiota interactions can inform drug posology and elucidate mechanisms of drug disposition.
\end{abstract}

\section{INTRODUCTION}

Pharmacokinetics (PK) of some orally administered medications are partially modulated by commensal organisms in the gut via microbial expression of drug-metabolizing enzymes, production of interfering substances, or effects on drug absorption. ${ }^{1-4}$ The antimalarial drug lumefantrine (LF), an arylamino alcohol, exhibits highly variable interindividual $P K$ with up to 16-fold differences in exposure. ${ }^{5}$ Variation is due in part to irregular absorption which is known to be mitigated by coadministration with fatty food. We hypothesized that the intestinal microbiome may play an additional, influential role in LF disposition.

Four cohorts ( $n=6$ per cohort) of 6-week-old female C57BL $6 \mathrm{~N}$ mice from three vendors $(\mathrm{E}=$ Envigo, $\mathrm{C}=$ Charles River, and $\mathrm{T}=$ Taconic Biosciences) and C57BL/6J mice from a fourth vendor ( $\mathrm{J}=$ Jackson Laboratories) previously shown to harbor distinct enterotypes ${ }^{6,7}$ were administered LF $(150 \mu \mathrm{g} / \mathrm{g})$ coformulated with artemether $(25 \mu \mathrm{g} / \mathrm{g})$ in $100 \%$ olive oil by oral gavage. Mice were identically housed in specific pathogen-free conditions with a 12-hour light/dark cycle from 6:00 to 18:00 and 18:00 to 6:00 and free-fed National Institute of Health-31 rodent diet (modified open formula mouse/rat irradiated diet; Harlan 7,913; Envigo, Indianapolis, IN) with autoclaved nonacidified reverse osmosis water. Acclimation under identical conditions was for 1 week before experimentation. Experiments were in compliance with local and national regulations of laboratory animal welfare and approved by the University of Louisville Institutional Animal Care and Use Committee.

Pre-dose fecal pellets were flash-frozen in liquid nitrogen and stored at $-80^{\circ} \mathrm{C}$. DNA extraction was performed with the

\footnotetext{
*Address correspondence to Matthew M. Ippolito, Johns Hopkins University School of Medicine, 725 N. Wolfe St. Ste. 211, Baltimore, MD 21205 or Nathan W. Schmidt, Department of Pediatrics, Ryan White Center for Pediatric Infectious Diseases and Global Health, Indiana University School of Medicine, 1044 West Walnut St. R4 470, Indianapolis, IN 46202, E-mails: mippolito@jhu.edu or nwschmid@ iu.edu.

†These authors contributed equally to this work.
}

QIAamp PowerFecal DNA kit (QIAGEN, Germantown, MD). Sequencing was performed by Washington University in the St. Louis Genome Technology Access Center using the multiple $16 S$ variable region species-level identification and analysis pipeline. ${ }^{8}$ Taxa plots, $\alpha$ diversity, and weighted UniFrac $\beta$ diversity were generated using QIIME version 1.9 (Knight Lab, University of Colorado, Boulder, CO) from cumulative sum scaling normalized operational taxonomic unit tables. ${ }^{9,10}$ One mouse each in cohorts $C$ and $T$ was excluded because of low $\alpha$ diversity. Bacterial community diversity was compared by oneway analysis of variance using Tukey's multiple comparisons test with a two-sided $\alpha$ of 0.05 in GraphPad Prism 7 (GraphPad Software, La Jolla, CA). Complete-linkage clustering on the weighted UniFrac distances was implemented using the "hclust" function in R version 3.6.3 (R Foundation for Statistical Computing, Vienna, Austria).

Retro-orbital whole blood $(50 \mu \mathrm{L})$ was collected pre-dose and post-dose according to intensive or sparse sampling schedules. Two mice from each cohort were sampled intensively at $0,0.25$, $0.5,1.5,10$, and 24 hours. One mouse from cohort $E$ was not sampled post-dose. The remaining were sampled at 0, 0.25, 0.5, and 10 hours or $0,0.2,1.5$, and 24 hours. Samples were collected in non-heparinized glass capillary tubes and placed immediately into $1.5-\mathrm{mL}$ tubes on ice, allowed to clot, then centrifuged at $13,000 \mathrm{rpm}(15,870 \times \mathrm{g})$ for $10 \mathrm{~m}$ at $4^{\circ} \mathrm{C}$. Serum was stored at $-80^{\circ} \mathrm{C}$. Pharmacokinetic sample preparation and quantitation were performed by protein precipitation followed by liquid chromatography-tandem mass spectrometry. The dynamic range was $50-20,000 \mathrm{ng} / \mathrm{mL}$ validated to an observed total assay coefficient of variation $<15 \%$ in all quality controls.

Maximal concentration $\left(C_{\max }\right)$ was the observed value at 10 hours for each individual mouse with available time points. Area under the drug concentration-time curve $\left(\mathrm{AUC}_{0-24}\right)$ was estimated using the linear-log trapezoidal method by noncompartmental analysis with sparse data methods, stratified by enterotype, using combined intensive and sparse PK data. Parameters were compared using Student's $t$-test with a twotailed $\alpha$. Pharmacokinetic analyses were performed in Phoenix 
WinNonlin (Certara, Princeton, NJ), and statistical analyses were carried out using Stata 14 (StataCorp, College Station, TX).

Mice from vendors $E$ and $C$ had significantly higher taxonomic abundance than those from $\mathrm{J}$ and $\mathrm{T}$ (Figure 1A), and weighted UniFrac $\beta$ diversity was lowest (i.e., similarity greatest) for $E$ and $C$ pairs, and $J$ and $T$ pairs (Figure 1B). The dendrogram of the UniFrac distance matrix revealed
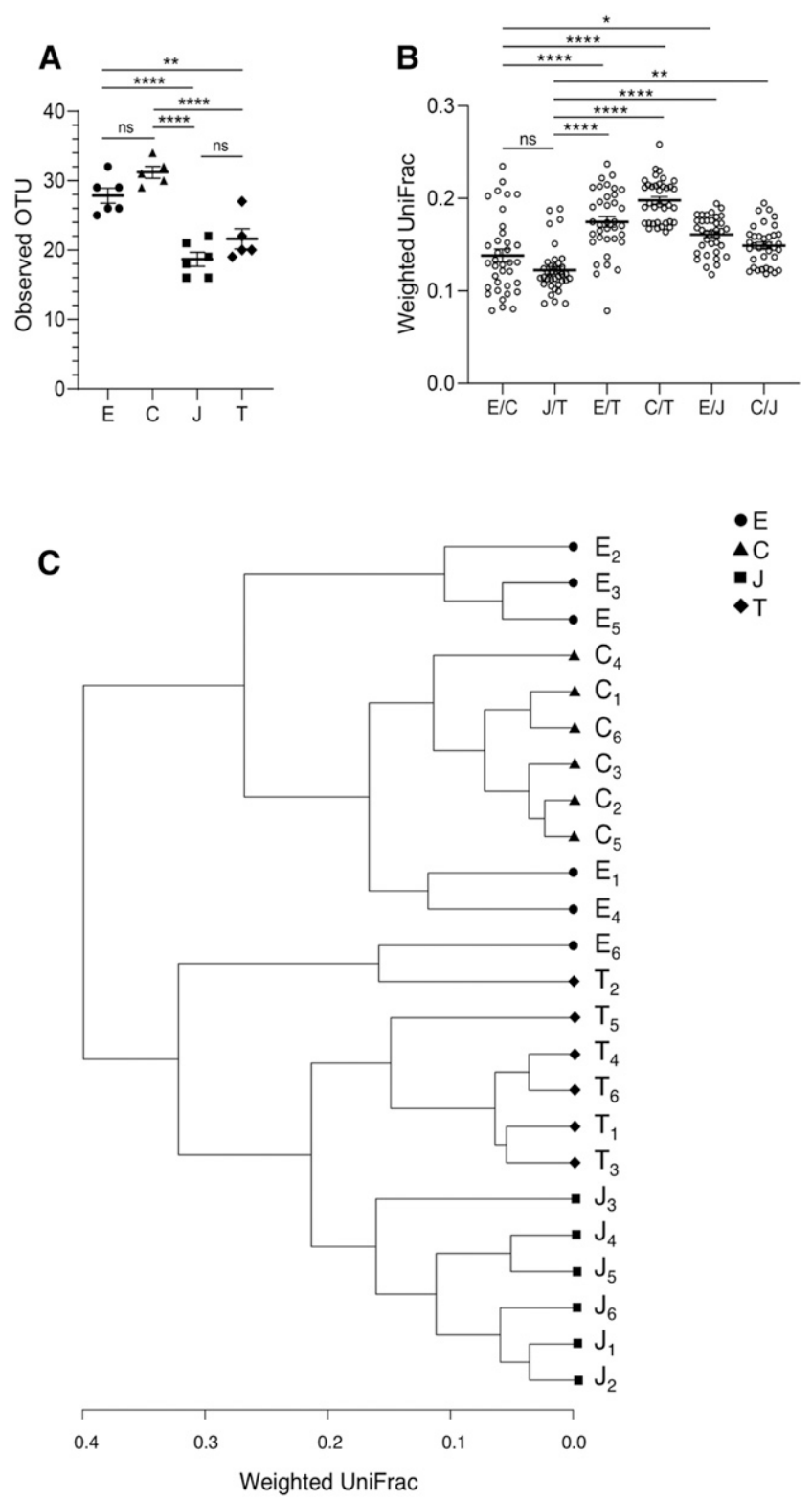

FIGURE 1. Computational analysis of microbiota data reveals partitioning of Envigo (E) with Charles River (C) cohorts and Jackson Laboratory $(\mathrm{J})$ with Taconic (T) cohorts into distinct clusters. (A) Alpha diversity of mice measured by observed operational taxonomic units (OTU). (B) Beta diversity comparisons between mice from different vendors measured by weighted UniFrac, with comparisons between enterotype clusters. Data are means and standard errors cumulative of two experiments for $n=5-6$ mice per cohort. Data were analyzed by one-way analysis of variance with Tukey's multiple comparisons test. (C) Dendrogram of the weighted UniFrac distance matrix reveals hierarchical clustering of $\mathrm{E} / \mathrm{C}$ and $\mathrm{J} / \mathrm{T}$ cohorts at the second and third bifurcations. Leaves represent individual mice. Clustering was independent of cage assignment. ${ }^{\star} P<0.05$, ${ }^{\star \star} P<0.01$, and ${ }^{\star \star \star \star} P<$ 0.0001. $\mathrm{ns}=$ not significant.
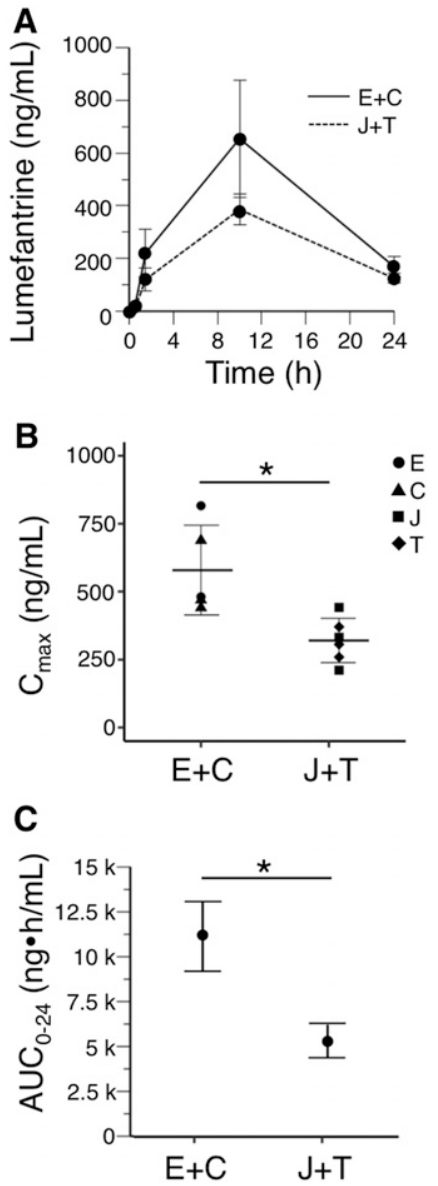

FIGURE 2. Lumefantrine pharmacokinetics are associated with gut microbiota composition in healthy mice. Mice were administered lumefantrine $(150 \mathrm{ug} / \mathrm{g})$ in combination with artemether $(25 \mathrm{ug} / \mathrm{g})$ by gavage at time 0 . (A) Concentration-time profiles stratified by enterotype. (B and $\mathbf{C}$ ) Mean and standard deviation peak concentrations $\left(C_{\max }\right)$ and areas under the drug concentration-time curve $\left(\mathrm{AUC}_{0-24}\right)$ were significantly higher in the Envigo (E) and Charles River (C) cohorts than those in the Jackson Laboratory $(J)$ and Taconic $(T)$ cohorts. $\mathrm{AUC}_{0-24}$ was measured using sparse data methods, therefore individual subject data are not computed. ${ }^{\star} P \leq 0.02$.

hierarchical clustering of the same pairs ( $E$ and $\mathrm{C}, \mathrm{J}$ and $\mathrm{T}$ ), overall conforming to two distinct fecal enterotypes. Mice from cohorts $E$ and $C$ had greater LF exposure than those from cohorts $\mathrm{J}$ and $\mathrm{T}$ (Figure 2). Means and standard deviations (SDs) of $C_{\max }$ were $660 \pm 220 \mathrm{ng} / \mathrm{mL}$ in cohorts $E$ and $C$ compared with $390 \pm 59 \mathrm{ng} / \mathrm{mL}$ in cohorts $\mathrm{J}$ and $\mathrm{T}(P=0.02)$, and the estimated $\mathrm{AUC}_{0-24}$ was $9,600 \pm 2,800$ compared with $5,800 \pm 810 \mathrm{ng} \times \mathrm{h} / \mathrm{mL}$, respectively $(P=0.01)$.

We measured the disposition of intragastrically dosed LF in isogenic mice with structurally distinc enterotypes and identified associations between drug PK and the gut microbial community structure. These findings support a possible contribution of the gut microbiota to the high intersubject variability frequently observed in PK studies of LF.

Direct and indirect gut microbiota effects on the absorption, distribution, metabolism, and/or elimination of LF may account for the observed differences in drug exposure. The excretion of LF relies on glucuronidation which is potentially impacted by bacterial commensal secretion of $\beta$-glucuronidases, and the drug's chemical structure renders it sensitive to 
bacterial lyases that cleave carbon-nitrogen bonds., 511,12 The biomass of the intestinal microbiota may make a discernible contribution to drug transit or sequestration, constituting a compartment into which small molecules might distribute. Indirect effects of gut commensals on drug transporter expression or luminal $\mathrm{pH}$ have been hypothesized to impede or facilitate absorption. 4

Limitations to this study include the relative paucity of PK time points which precluded reliable estimations of oral clearance or apparent volume of distribution, or the evaluation of the interaction between enterotype and individual PK parameters.

Acute and chronic malnutrition are common in patients with malaria, and the accompanying alterations to gut microbial communities $^{13,14}$ may affect drug PK. Antimalarial drug PK and pharmacodynamics differ between healthy and infected individuals ${ }^{5}$; gut microbiome differences are one potential contributor.

The characterization of gut microbial effects on PK of orally administered agents could inform drug posology and improve models of drug disposition by helping to account for interindividual variability. Future experiments that incorporate gnotobiotic controls and fecal transplantation, and studies in human subjects, could go further toward establishing causal and clinically relevant linkages between the gut microbiome and drug PK.

Received April 22, 2020. Accepted for publication May 28, 2020.

Published online July 6, 2020.

Acknowledgments: We thank Mary Barry for assisting with sample preparation for bioanalytical assays, and we thank Cynthia L. Sears, Craig W. Hendrix, Michelle A. Rudek, and Rahul Bakshi for their critical review of the draft manuscript.

Financial support: This work was supported by the National Institutes of Health (grant nos. K23Al139343 to M. M. I., Al123486 to N. W. S., and Al095453 to T. A. S.), Johns Hopkins Malaria Research Institute, Bloomberg Philanthropies, Burroughs Wellcome Fund-American Society of Tropical Medicine and Hygiene Postdoctoral Fellowship in Tropical Infectious Diseases, and the Sherrilyn and Ken Fisher Center for Environmental Infectious Diseases at the Johns Hopkins University School of Medicine (to M. M. I.).

Authors' addresses: Matthew M. Ippolito, Department of Medicine, Johns Hopkins University School of Medicine, Baltimore, MD, E-mail: mippolito@jhu.edu. Joshua E. Denny, Department of Microbiology and Immunology, University of Pennsylvania, Philadelphia, PA, E-mail: joshua.denny@pennmedicine.upenn.edu. Elizabeth Nenortas, Division of Clinical Pharmacology, Johns Hopkins University School of Medicine, Baltimore, MD, E-mail: enenort1@jhmi.edu. Theresa A. Shapiro, Johns Hopkins University School of Medicine, Baltimore, MD, E-mail: tshapiro@jhmi.edu. Nathan W. Schmidt, Ryan White
Pediatric Infectious Diseases and Global Health, and Department of Pediatrics, Indiana University School of Medicine, Indianapolis, IN, E-mail: nwschmid@iu.edu.

\section{REFERENCES}

1. Zimmermann $M$, Zimmermann-Kogadeeva $M$, Wegmann $R$, Goodman AL, 2019. Separating host and microbiome contributions to drug pharmacokinetics and toxicity. Science 363: eaat9931.

2. Kobashi K, Akao T, Hattori M, Namba T, 1992. Metabolism of drugs by intestinal bacteria. Bifidobacteria Microflora 11: 9-23.

3. Clayton TA, Baker D, Lindon JC, Everett JR, Nicholson JK, 2009. Pharmacometabonomic identification of a significant hostmicrobiome metabolic interaction affecting human drug metabolism. Proc Natl Acad Sci U S A 106: 14728-14733.

4. Matuskova Z, Anzenbacherova E, Vecera R, TlaskalovaHogenova H, Kolar M, Anzenbacher P, 2014. Administration of a probiotic can change drug pharmacokinetics: effect of E. coli Nissle 1917 on amidarone absorption in rats. PLoS One 9: e87150.

5. White NJ, van Vugt M, Ezzet F, 1999. Clinical pharmacokinetics and pharmacodynamics of artemether-lumefantrine. Clin Pharmacokinet 37: 105-125.

6. Denny JE, Powers JB, Castro HF, Zhang J, Joshi-Barve S, Campagna SR, Schmidt NW, 2019. Differential sensitivity to Plasmodium yoelii infection in C57BL/6 mice impacts gut-liver axis homeostasis. Sci Rep 9: 3472.

7. Villarino NF, LeCleir GR, Denny JE, Dearth SP, Harding CL, Sloan SS, Gribble JL, Campagna SR, Wilhelm SW, Schmidt NW, 2016. Composition of the gut microbiota modulates the severity of malaria. Proc Natl Acad Sci U S A 113: 2235-2240.

8. Schriefer AE, Cliften PF, Hibberd MC, Sawyer C, Brown-Kennerly V, Burcea L, Klotz E, Crosby SD, Gordon JI, Head RD, 2018. A multi-amplicon 16S rRNA sequencing and analysis method for improved taxonomic profiling of bacterial communities. $J$ Microbiol Methods 154: 6-13.

9. Caporaso JG et al., 2010. QIIME allows analysis of highthroughput community sequencing data. Nat Methods 7: 335-336.

10. Lozupone C, Knight R, 2005. UniFrac: a new phylogenetic method for comparing microbial communities. Appl Environ Microbiol 71: 8228-8235.

11. Bindschedler MDP et al., 1996. Comparative bioavailability of benflutemol after administration of single oral doses of coartemether under fed and fasted conditions to healthy subjects [abstract]. XIVth International Congress for Tropical Medicine and Malaria. Brisbane, Australia: Australasian Society for Infectious Diseases.

12. Biernat KA et al., 2019. Structure, function, and inhibition of drug reactivating human gut microbial beta-glucuronidases. Sci Rep 9: 825.

13. Smith $\mathrm{Ml}$ et al., 2013. Gut microbiomes of Malawian twin pairs discordant for kwashiorkor. Science 339: 548-554.

14. Kane AV, Dinh DM, Ward HD, 2015. Childhood malnutrition and the intestinal microbiome. Pediatr Res 77: 256-262. 